\title{
Rheological and textural studies of fresh and freeze-thawed native sago starch-sugar gels. I. Optimisation using response surface methodology.
}

\begin{abstract}
A three-factor-three-level Box-Behnken design was adopted to study the simultaneous effects of two compositional variables (6-8\% sago starch and 25-35\% sugar) and one processing variable (shearing speed of mixer at 20-50 rpm) on textural and rheological properties of gels. Analysis of variance (ANOVA) was performed to evaluate the potential interactive and quadratic effects between these variables. Sago starch and sugar levels both increased gel stiffness and viscoelasticity. Shearing, on the other hand, reduced gel stiffness and viscoelasticity. Ridge analysis was performed to estimate the values of these variables which maximised and minimised the textural parameters of hardness, gumminess, resilience, cohesiveness, and springiness. Pearson correlations among various rheological and textural properties of gels were studied. The processing conditions that contributed to an optimum gel setting were found at sago starch of level of $7.69 \%$, sugar of $30.29 \%$, and shearing speed of $45.86 \mathrm{rpm}$.
\end{abstract}

Keyword: RSM; Sago starch; Gel; Texture; Rheology. 